\title{
Método para Construção de Ontologia de Pictogramas para Sistemas de Comunicação Alternativa
}

\author{
Cláudia C. C. Pérez ${ }^{1}$, Liliana Marina Passerino ${ }^{1}$, Bianca Peixoto ${ }^{2}$ \\ ${ }^{1}$ Programa de Pós-Graduação em Informática na Educação - Universidade Federal do \\ Rio Grande do Sul (UFRGS) \\ ${ }^{2}$ Programa de Pós-Graduação em Educação - Universidade Federal do Rio Grande do \\ Sul (UFRGS) \\ \{claudia.perez, lpasserino, bnpeixoto\}@gmail.com
}

\begin{abstract}
Resumo. Esse artigo tem como objetivo apresentar uma método para construção de ontologia de pictogramas para sistemas de Comunicação Alternativa como uma forma de implementar uma busca por pictogramas utilizando contextos semânticos. Será relatado como a ontologia de pictogramas foi construída e seus resultados e, por fim, serão elencados seus usos futuros em sistemas de Comunicação Alternativa para construção de pranchas de comunicação. A representação de conhecimento com a ontologia de pictogramas compõe a busca semântica do sistema SCALA.
\end{abstract}

Palavras-chaves: ontologia, pictogramas, Comunicação Alternativa.

\section{Method for the Construction of Pictograms Ontology for Alternative Communication Systems}

\begin{abstract}
This paper aims to present a method for the construction of pictograms ontology for Alternative Communication systems as a way to implement a search for pictograms using semantic contexts. It will be reported how the ontology of pictograms was built and its results and, finally, will be listed its future uses in alternative communication systems for construction of communication boards. The knowledge representation with the ontology of pictograms composes the semantic search of the system SCALA.
\end{abstract}

Keywords: ontology, pictograms, Alternative Communication.

\section{Introdução}

A Comunicação Alternativa (CA) é uma das áreas da Tecnologia Assistiva (TA) que se dedica a desenvolver símbolos (pictogramas), recursos, técnicas e estratégias utilizadas para favorecer ou incentivar outra forma de comunicação de pessoas com distúrbios físicos ou mentais, os quais impossibilitam (ou dificultam) o desenvolvimento da fala ou da escrita (PELOSI, 2015). Um tipo de recurso de CA são as pranchas de comunicação, compostas por pictogramas selecionados de acordo com as necessidades e objetivos de comunicação. As pranchas podem dispor de fotografias, desenhos, imagens confeccionadas, legendas, alfabeto, números e quaisquer outros elementos que se façam necessários. Estudos sobre esses recursos envolvem pesquisas de sistemas de CA para computador, web, tablets, e dispositivos móveis. Sistema de CA como o SCALA Sistema de Comunicação Alternativa para o Letramento de pessoas com Autismo, desenvolvido para a web e tablet (PASSERINO, 2010; PASSERINO, 2013); o AraBoard (BALDASSARRI, 2014) e o BoardMaker (MAYER JOHNSON, 2017) 
disponíveis para computador, smartphone ou tablete e o Prancha Fácil (PELOSI, 2015) entre outros.

A maioria dos sistemas de CA é organizada por categorias (substantivos, qualidades, verbos) úteis para a busca manual das imagens e que exige um conhecimento prévio do que se deseja procurar. Desta forma, sistemas de CA estabelecem como requisito certa capacidade linguística do usuário em saber de antemão que tipo de elemento o pictograma representa (por exemplo, um adjetivo). Esses sistemas também não exploram ou descrevem contextos de uso. O que representa uma limitação comum a todos é a não existência de busca semântica por pictogramas, ficando essa tarefa por conta das categorias de imagens predefinidas ou criadas pelos usuários na interação com o sistema.

No que se refere à construção de pranchas de comunicação, observa-se que a ideia de busca de imagens por palavra-chave ou seleção manual de imagem em categorias não é nova, uma vez que existem diversas soluções tanto nacionais como internacionais na área de CA que possuem essas possibilidades. Nossa proposta, entretanto, diferencia-se em sua abordagem, pois apresenta a busca semântica de pictogramas. Sendo assim, o que será apresentado no presente artigo é um recorte de pesquisa sobre a construção de uma ontologia de pictogramas integrada a uma ferramenta de busca semântica de pictogramas em sistema de CA. Tendo como objetivo apresentar uma método de construção de uma ontologia específica para pictogramas de CA, mapeados com conceitos do contexto, para compor a base de conhecimento do sistema de busca semântica de pictogramas aplicados na construção de pranchas de comunicação alternativa. A ferramenta de busca semântica é formada pela busca simples da palavra-chave e pela busca avançada com o contexto semântico.

O sistema de CA que serviu como base para este estudo é o SCALA que funciona tanto na web quanto em dispositivos móveis com sistema Android, atualmente com 3971 usuários cadastrados, e um banco de pictogramas com 4232 símbolos e 24 cenários. $\mathrm{O}$ artigo encontra-se organizado nas seguintes seções: no primeiro tópico serão apresentadas questões conceituais sobre ontologias e, em seguida, será apresentado o método para construção de ontologia de pictogramas.

\section{Método para construção de ontologia de pictogramas}

Uma definição de ontologia muito citada é a de Gruber (1996, p. 2): "uma ontologia é uma especificação formal, explícita de uma conceitualização compartilhada". Onde podemos entender: "conceitualização" como uma visão abstrata e simplificada do mundo que se deseja representar; "formal" se refere ao fato de que a ontologia deveria ser legível por máquina; "explícita" significa que o tipo de conceitos utilizados, e as restrições na sua utilização, são definidos explicitamente; e "compartilhada" indica que a ontologia deveria capturar conhecimento consensual aceito pelas comunidades que irão usar a ontologia como referência. Ontologias podem ser utilizadas em várias áreas tais como: recuperação de informações na internet, processamento de linguagem natural, gestão do conhecimento, web semântica e educação.

Existem várias propostas na literatura sobre como construir uma ontologia: Enterprise (USCHOLD e KING, 1995); Methontology (FERNANDEZ, GOMEZPEREZ e JURISTO, 1997); e o Método 101 (NOY e McGUINNESS, 2001). Nesse trabalho, foram reunidas características da Methontology e do Método 101, por essas metodologias considerarem questões importantes na construção de ontologia de 
pictogramas: Qual o domínio que a ontologia deve cobrir? Para o que a ontologia será usada? A que tipos de questões as informações contidas na ontologia devem responder? Quem usará e manterá a ontologia? (FERNANDEZ, 1997; NOY, 2001).

A ontologia desta pesquisa tem como domínio o contexto higiene pessoal de crianças entre 4 e 7 anos. Justifica-se a escolha dessa faixa etária em função da importância da intervenção precoce. Aos três anos de idade, as crianças estão desenvolvendo autonomia incluindo atividades como higiene das mãos, desfralde, escovação e banho, e, também sua inclusão na escola. Ontologia usada em sistema de $\mathrm{CA}$, por adultos que auxiliam crianças a se comunicar, através das pranchas de comunicação. As informações contidas na ontologia respondem questões norteadoras do tipo: quais objetos e ações são utilizados na escovação de dentes, para tomar banho, para fazer xixi, para lavar as mãos, entre outros.

\subsection{Seleção de Pictogramas}

A primeira etapa do método para a construção da ontologia foi a seleção de pictogramas de contexto. O estudo foi centrado no ambiente familiar e escolar, sobretudo no contexto de uso do banheiro da escola, da casa e das atividades de higiene pessoal e crianças entre 4 e 7 anos. O primeiro passo consistiu no levantamento de pictogramas do SCALA e Portal ARASAAC, dentro do contexto e público alvo acima referidos. A

partir das questões norteadoras foram adotadas duas estratégias de pesquisa por pictogramas:

a) a primeira estratégia adotada é escolher a ação de higiene e em seguida listar os elementos necessários para sua realização. Por exemplo: a criança deseja lavar o rosto. Para tanto, serão necessários: o uso da pia, da água, da torneira (abrir e fechar), sabonete (para ensaboar e esfregar o rosto), e toalha (para secar o rosto e as mãos).

b) a segunda estratégia adotada é escolher o objeto e em seguida listar as ações que podem ser realizadas com seu uso. Por exemplo: a criança busca a escova de dente. Com esse objeto, ela pode escovar os dentes. Porém para a ação de escovar os dentes, também serão necessários: pasta de dente (apertar pasta de dente, colocar sobre a escova de dente), fio dental (puxar, cortar e passar fio dental nos dentes), antisséptico bucal (bochechar), pia, torneira (abrir e fechar), água e toalha (para secar as mãos e a boca).

Figura 1. Exemplo de pictogramas selecionados

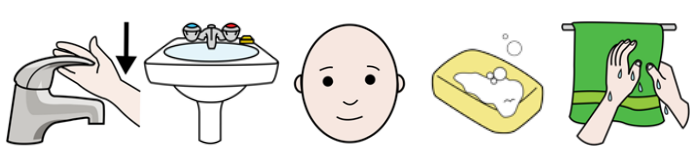

Fonte: Elaborado pelos autores

No SCALA, os pictogramas que envolvem atividades de higiene pessoal foram encontrados nas categorias Objetos e Ações, sendo selecionados inicialmente 98 pictogramas da categoria Objetos e 47 pictogramas da categoria Ações. Na pesquisa do portal ARASAAC foram acrescentados ao conjunto 9 pictogramas caracterizados por ações e seis por objetos. Somando-se a este novo conjunto, três pictogramas de objetos e 11 de ações foram criados pelo designer do projeto SCALA, totalizando inicialmente 107 pictogramas da categoria Objetos e 67 da categoria Ações. 


\subsection{Mapeamento do Contexto Semântico}

A segunda etapa do método envolveu o mapeamento do contexto semântico com a representação gráfica das relações conceituais existente entre os pictogramas, conforme as questões norteadoras da ontologia. As relações conceituais estabelecem o tipo de relacionamento existente entre os conceitos, e podem apontar para outros termos que se relacionam ao conceito. Explicitando-se as relações entre os conceitos, garante-se a consistência da estrutura terminológica do instrumento utilizado para a organização do domínio, como na ontologia, e sua formalização facilita a inserção dos conceitos no sistema e sua recuperação pela máquina. Para a representação gráfica do mapeamento utilizou-se a ferramenta Cmap Tools (IHMConcept, 2016). Na ferramenta, os conceitos são colocados em retângulos (com ou sem cantos arredondados), que por sua vez são interligados por linhas rotuladas (elipses) que possuem a descrição dessa relação. É a criação desta unidade semântica que tem maior valor, ou seja, que dá a razão de ser de um mapa conceitual.

Nessa etapa do mapeamento do contexto semântico, a modelagem com os mapas conceituais permitiu a representação gráfica básica das relações conceituais do tipo lógicas, hierárquicas, partitivas, de oposição e funcionais dentro de um domínio (DAHLBERG, 1978), existentes entre os pictogramas selecionados na etapa anterior.

No mapeamento do contexto, os pictogramas da categoria Objetos correspondem aos conceitos do mapa conceitual, e os pictogramas da categoria Ação representam, na maioria das vezes, as relações entre os conceitos. Na falta de um pictograma da categoria Ação para as relações conceituais, a inclusão de relações "contém", por exemplo.O resultado foi a modelagem básica das relações conceituais estabelecidas por triplas do tipo <conceito - relação - conceito> ou <objeto - ação - objeto>, exemplo:

$<$ Toalha - Secar - Mãos>

$<$ Dedos - Contém - Unhas >

Uma relação (ação) pode ligar um ou vários conceitos (objetos), exemplo:

$<$ Toalha - Secar - Mão>

$<$ Toalha - Secar - Pés>

$<$ Sabonete - Tipo - Sabonete_barra $>$

$<$ Sabonete - Tipo - Sabonete_líquido>

No total, foram criados 13 mapas conceituais do contexto higiene pessoal, com a ferramenta CMap Tools, na etapa do mapeamento semântico, com o objetivo de identificar e avaliar as relações semânticas entre os pictogramas selecionados, verificar a necessidade de inclusão de mais pictogramas existentes na base de pictogramas do SCALA ou identificar a produção ou adequação de pictogramas.

Figura 2. Mapa Conceitual - lavar o rosto

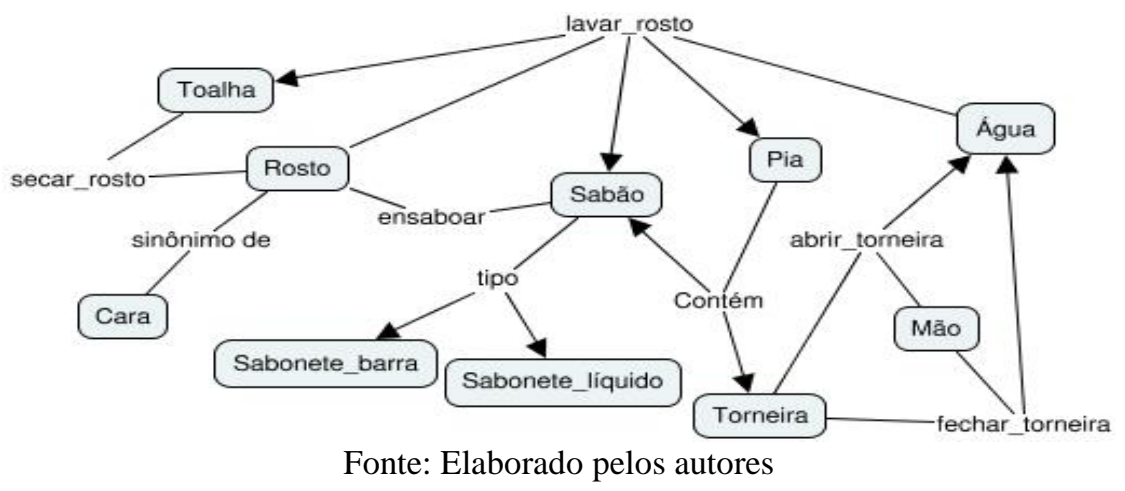


A Figura 2 ilustra o mapa conceitual construído na etapa mapeamento do contexto, com os pictogramas selecionados das categorias Objetos e Ações, para a atividade "lavar o rosto" com pictogramas: rosto, cara, mão, pia, torneira, água, sabão, sabonete_barra, sabonete_líquido, toalha, ensaboar, abrir e fechar torneira, lavar e secar o rosto.

Este tipo de modelagem permitiu definir o domínio, mapear conceitos e relações da ontologia para a busca semântica no SCALA.

Para testar e analisar os resultados obtidos foi realizado um Quase Experimento inicial que envolveu dois testes. Os testes foram realizados com três alunos de doutorado do grupo de pesquisa TEIAS ${ }^{1}$ - Tecnologia em Educação para Inclusão e Aprendizagem em Sociedade, desses alunos, dois pesquisam sobre TA e um trabalha com CA e autismo, tendo como objetivos: a) verificar a adequação dos pictogramas ao domínio selecionado; b) avaliar as relações semânticas encontradas entre os pictogramas; c) extrair novos contextos de uso dos pictogramas junto aos sujeitos pesquisados.

O Quase Experimento foi escolhido como método de pesquisa, por estar em um ponto intermediário de controle do experimento. A palavra "quase" no campo do design experimental é utilizada para se referir aos designs os quais o pesquisador não pode obter um controle total sobre o procedimento do experimento ou não pode designar aleatoriamente os sujeitos pesquisados para os procedimentos. $\mathrm{O}$ pesquisador recorre aos quase experimentos quando se interessa pela validação externa dos resultados ou quando, por várias razões, não consegue realizar experimentos reais (VIEYTES, 2004).

$\mathrm{O}$ Quase Experimento foi realizado individualmente, em momentos e locais distintos, tendo duração média de 1 hora. Os dois testes do Quase Experimento contaram com o auxílio de uma bolsista de iniciação científica, ficando sob sua responsabilidade fazer os registros com filmagem, fotos e anotações, durante as interações do pesquisador com os sujeitos pesquisados, além de analisar o material obtido através dos registros. Para a análise e avaliação levou-se em consideração: duração, carga cognitiva, qualidade das instruções e estrutura, avaliação do sujeito sobre o quase experimento.

Utilizou-se um conjunto de 174 pictogramas coloridos impressos recortados e cobertos por plástico, medindo $6 \mathrm{~cm} \times 4 \mathrm{~cm}$. O Teste 1 , com o sujeito da pesquisa classificando os pictogramas, selecionados no estudo do mapeamento, nas categorias "Muito uso", "Médio uso", "Pouco uso" e separando os pictogramas que o sujeito acredita não se aplicarem para o domínio higiene pessoal com crianças de quatro a sete anos. Além disso, foram dispostas, nas mesas de apoio, cartões de papel indicando a classificação de uso dos conceitos: Muito Uso, Médio Uso e Pouco Uso. Informa-se, ainda, que ao longo do Teste 1, a categoria "Não se Aplica" foi incluída para designar os pictogramas com legenda e/ou desenho insatisfatório, pictogramas (imagens) repetidos ou que representavam ações/objetos de mesmo significado, com uso inadequado para a faixa etária estabelecida ou, ainda, objetos que seriam manuseados pelos pais ou responsáveis, e não, exatamente, pelas crianças.

O Teste 2, ao sujeito pesquisado, propor a seleção e ordenação dos pictogramas para construção de pranchas de comunicação das seguintes ações/tarefas: escovar os

${ }^{1}$ http://www.ufrgs.br/teias/ 
dentes, fazer xixi, fazer cocô, lavar as mãos, lavar o rosto, tomar banho e pentear cabelo.

A análise qualitativa e quantitativa dos testes mostrou que a pesquisa elencou um número maior de objetos e ações que os sujeitos. Concluiu-se que a ontologia abrangeu um grande número de conceitos e ações, garantindo um quantitativo relevante de elementos para cada contexto de uso da higiene pessoal. Após o mapeamento do contexto semântico e do primeiro Quase Experimento, foram incluídos mais 49 da base de pictogramas do SCALA, considerados relevantes ao contexto higiene pessoal, e totalizando 223 pictogramas.

\subsection{Construção da ontologia}

Nesta etapa foram considerados os resultados obtidos nas etapas anteriores. Para o projeto de construção da ontologia foram reunidas características das metodologias Methontology e do Método 101 e o ambiente Protégé. Na sequência, para a construção da ontologia o estudo das bases de dados de conhecimento linguístico WordNet.PT e PAPEL. A WordNet.PT é uma base de dados de conhecimento linguístico de português, desenvolvida no Centro de Linguística da Universidade de Lisboa. Uma Wordnet é uma rede léxico-conceitual estruturada em torno de um conjunto de relações e tenta corresponder à organização mental do significado lexical. O PAPEL - Palavras Associadas Porto Editora - Linguateca - é uma rede lexical pública para o português, extraída de forma automática a partir do Dicionário PRO da Língua Portuguesa da Porto Editora.

A sequência de etapas para a construção de uma ontologia podem ser resumidas em dois tópicos importantes: (1) Domínio da ontologia - determinar o domínio, o reúso e a lista de termos importantes da ontologia. Nessa etapa foi realizado o estudo de caso com os Mapas Conceituais e a possibilidade de organizar conceitos e relações conceituais para a ontologia, denominado mapeamento semântico. Reúso de conceitos e relações de bases de dados de conhecimento linguístico WordNet.PT e PAPEL; (2) Implementação da ontologia - identificação das classes, relações, propriedades e indivíduos da ontologia no Protégé, e da linguagem RDF/XML de representação para codificação. Avaliação da ontologia que foi feita durante todo o ciclo de vida do processo, assegurando a sua eficiência no final.

A ontologia representa a base das relações conceituais para que se possa realizar uma busca avançada (semântica) no banco de imagens do SCALA.

Figura 3 - Sistema SCALAi*

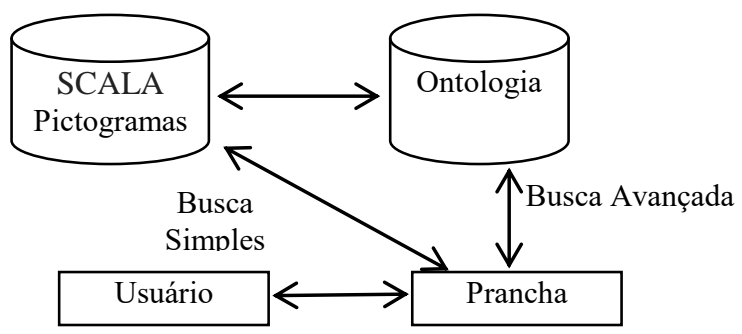

Fonte: Elaborado pelos autores

A seguir, é apresentado o estudo de reúso de conceitos e relações conceituais das bases de dados de conhecimento linguístico WordNet.PT e PAPEL, e na sequência, a codificação da ontologia com o uso da ferramenta Protégé. Foram pesquisados na WordNet.PT e PAPEL todos os conceitos representados pelos pictogramas 
selecionados, para avaliar as relações conceituais encontradas no mapeamento de contexto de uso em comparação com as das bases lexicais. A WordNet.PT contém cerca de 19000 expressões, repartidas por vários campos semânticos, e inclui expressões relativas a vários subdomínios, tais como arte, geografia, instituições, saúde, seres vivos, transportes e vestuário.

A consulta na base lexical apresenta como resultado a estrutura de relações conceituais, conforme os exemplos a seguir:

Toalha de rosto

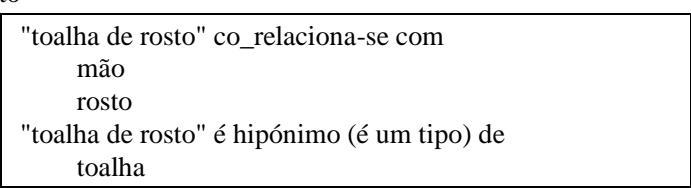

Este estudo possibilitou avaliar e acrescentar conceitos e relações conceituais identificadas na etapa de mapeamento do contexto semântico. Além disso, compor o formalismo necessário para a construção da ontologia no que se refere ao nome e significado das relações entre conceitos da ontologia.

Com base nas relações e respectivas descrições definidas nas ontologias WordNet.PT e PAPEL, a ontologia do SCALAi* considerou o conjunto que segue: Parte_De (WordNet), Tem_Como_Parte (WordNet.PT), Co_relaciona_se_Com (WordNet.PT), Hiponimo (WordNet.PT), Lugar_Para (WordNet.PT), Sinonimo_De (WordNet.PT), Antonimo_De (Papel), Finalidade_De (Papel) e Usado_Em_Acao (Papel). A seguir, é apresentada a codificação da ontologia com o uso da ferramenta Protégé.

\subsection{Ontologia no Protégé}

Nessa etapa, foi utilizado o ambiente Protégé para a continuação do desenvolvimento da ontologia com o formalismo das classes, relacionamentos e indivíduos, resultantes das etapas anteriores de mapeamento do contexto semântico e construção da ontologia. Conforme já apresentado, essa ontologia define a terminologia principal adotada na busca avançada/semântica do SCALAi*. No Protégé foram utilizadas as guias Classes, Object Properties e Individuals. A guia Classes permite a criação e manutenção de classes. A guia Object Properties é usada para a criação e manutenção das propriedades, que são relação binária entre indivíduos, ou seja, relaciona um indivíduo a outro indivíduo. A guia Individuals cria as instâncias das classes, ou seja, a população de indivíduos da ontologia.

O conjunto de classes definidas para este contexto, a partir da classe Pictograma, foi: Ação, Local, Objeto, Pessoa. Dessas classes, foram definidos 223 conceitos e 1383 relações. Também foram criadas subclasses, como por exemplo, a classe Escova e as subclasses Escova de Cabelo, de Dentes e de Unhas. As classes e subclasses estão associadas aos pictogramas do banco de imagens do SCALA.

As propriedades das classes da ontologia são organizadas no Protégé na guia Object Properties. Essas propriedades da ontologia foram inseridas no projeto conforme o conjunto de relações conceituais identificadas na WordNet.PT e PAPEL. Foram utilizadas as Annotation (Comment) para inserir o significado de cada propriedade e Description (Inverse of) usado para vincular metadados às relações (Object Properties), conforme Figura 4, quadrantes a) classes e subclasses e b) Object Properties. 
Figura 4 - Protegé - Classes e subclasses - Object Properties
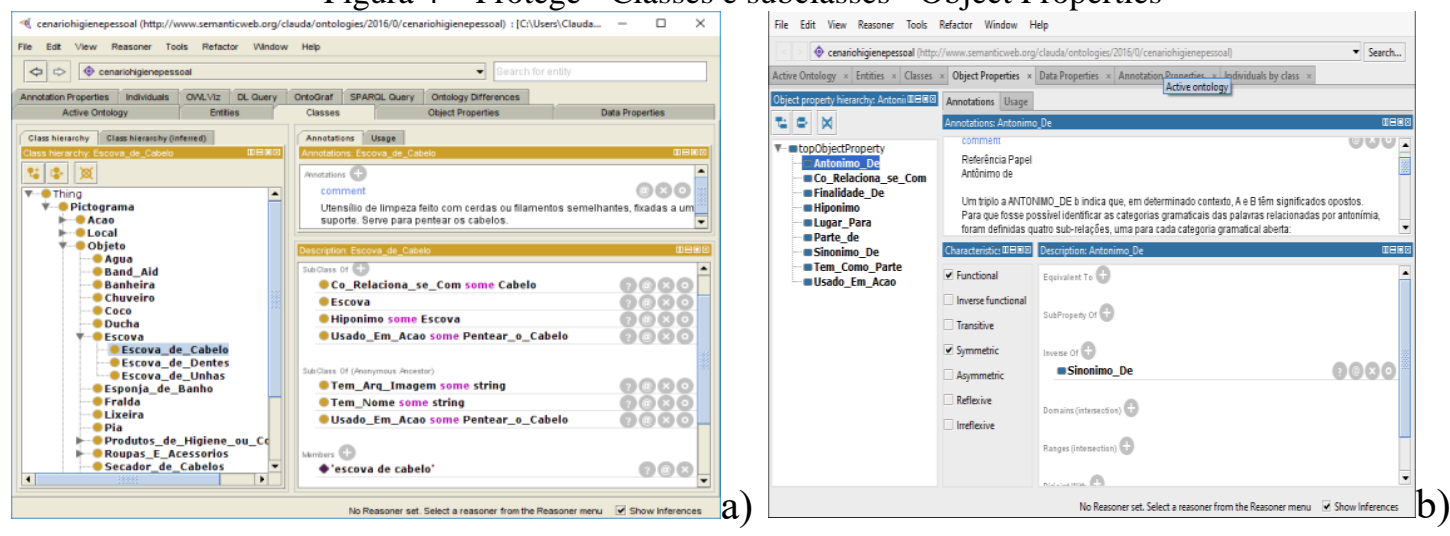

Fonte: Elaborado pelos autores

Domínio e subdomínio de propriedades (relações) podem ser definidos entre conceitos. Não são interpretados como restrições e, sim, como axiomas. Os axiomas consistem quando múltiplas classes compõem o subdomínio ou o domínio de uma relação, pois só assim é considerada a união dessas classes. Automaticamente o Protégé defini propriedades entre conceitos, com a descrição SubClass Of (Anonymous Ancestor).

As instâncias foram criadas na guia Individuals e estão relacionadas com as classes, propriedades e atributos. Os atributos de uma instância são definidos no Protégé na guia Data Properties. Para a ontologia foram definidos os atributos: Tem_Nome (nome da imagem que será procurada na ontologia) e Tem_Arq_Img (nome do arquivo de imagem .png). É importante frisar que estas instâncias são obrigatórias, fazendo parte do escopo da ontologia.

\subsection{Generalização para construção de ontologia de pictogramas}

O método de construção da ontologia de pictogramas, com as etapas e procedimentos de seleção dos pictogramas, mapeamento do contexto semântico e construção da ontologia, resultou a proposição de um fluxo geral de construção da ontologia de pictogramas para outros contextos de uso/semânticos. O SCALAi* poderá continuar em permanente atualização para outros contextos como: lazer, alimentação, escola, entre outros.

Figura 5 - Método para construção de ontologia de pictogramas

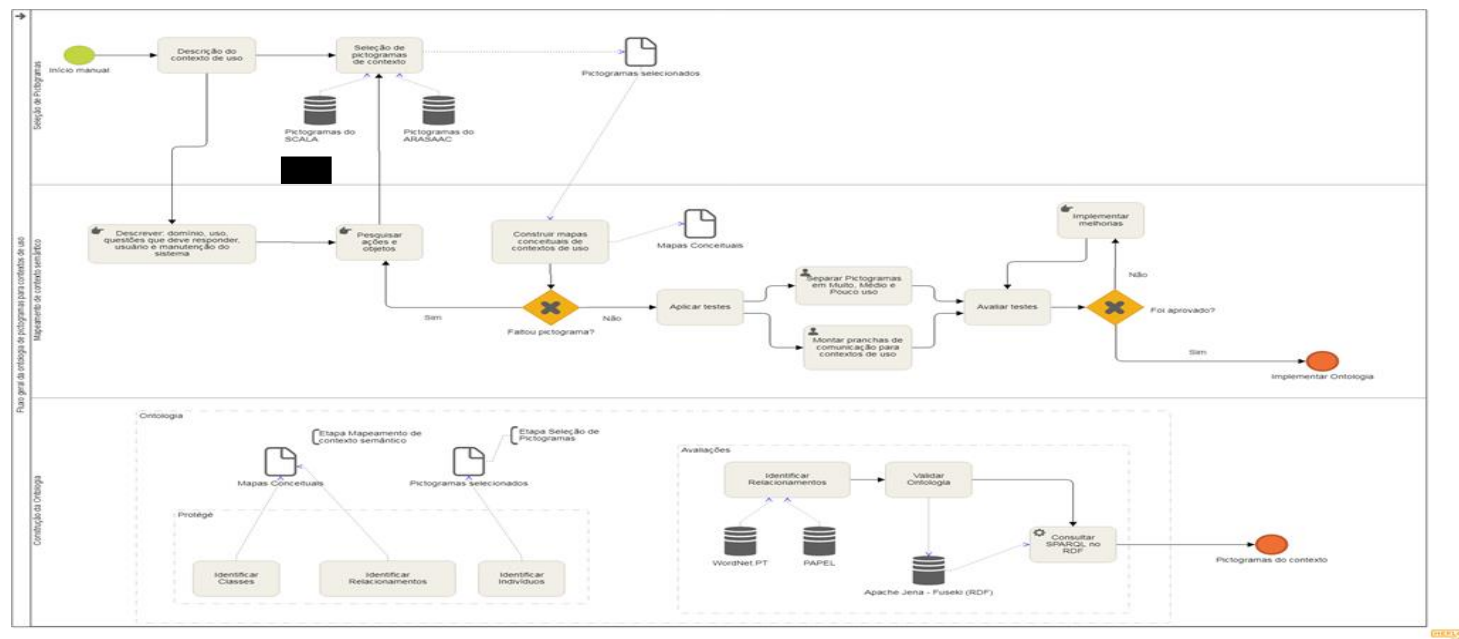

Fonte: Elaborado pelos autores 


\section{Considerações finais}

Para a pesquisa sobre a integração de ontologia em ferramenta de busca semântica de pictogramas em sistema de Comunicação Alternativa, foi apresentada a ontologia com conceitos de domínio específico do SCALA para responder questões relacionadas ao contexto higiene pessoal no ambiente domiciliar e escolar, integrada a ferramenta de busca semântica. Para compor os conceitos da ontologia, foram pesquisados pictogramas do SCALA e do ARASAAC. As estratégias de pesquisa por pictogramas foram: primeiro pela escolha da ação e segundo pelos elementos necessários para a realização da ação, resultando ao longo da pesquisa em um total de 223 pictogramas.

A modelagem com mapas conceituais permitiu a representação gráfica básica das relações conceituais do tipo lógicas, hierárquicas, partitivas, de oposição e funcionais dentro de um domínio. Para testar e analisar os resultados obtidos, foi realizado um experimento que envolveu dois testes, com três sujeitos do grupo de pesquisa TEIAS. Além dos mapas conceituais, o reúso de conceitos e relações de bases de dados de conhecimento linguístico WordNet.PT e PAPEL.

Para o projeto de construção da ontologia, foram reunidas características das metodologias Methontology e do Método 101, o ambiente Protégé (identificação das classes, relações, propriedades e indivíduos) e da linguagem RDF/XML de representação para codificação. A avaliação da ontologia foi realizada durante todo o ciclo de vida do processo, assegurando a sua eficiência no final.

Ressalta-se que o método contribuiu para a modelagem de uma ontologia de pictogramas para sistemas de CA, a proposição de um fluxo geral para a construção de ontologia de pictogramas para outros contextos e uma arquitetura para $\mathrm{o}$ desenvolvimento de ferramenta de busca avançada (sistema semântico) em sistema de CA, com a utilização de recursos tecnológicos.

\section{Agradecimentos}

Agradecemos à CAPES e ao CNPQ pelo financiamento de bolsistas e pesquisadores.

\section{Referências}

BALDASSARRI, Sandra; et al. (2014) AraBoard: A Multiplatform Alternative and Augmentative Communication Tool. Procedia Computer Science 27. 197 - 206.(in press in Procedia Computer Science - Journal - Elsevier) https://doi.org/10.1016/j.procs.2014.02.023. Acesso em mar.2018.

DAHLBERG, I. Teoria do conceito. (1978) Ciência da informação, v. 7, n. 2, p. 101107.

FERNANDEZ, M.; GÓMEZ-PÉREZ, A.; JURISTO, H. (1997) Methontology: from ontological art towards ontological engineering. AAAI Technical Report SS-97-06. Disponível em: <http://oa.upm.es/5484/1/METHONTOLOGY_.pdf> Acesso em nov.2017.

GRUBER, T. What is na ontology?, 1996. Handbook on Ontologies, International Handbooks. Springer, Berlin, Heidelberg. Disponível em:< http://iaoa.org/isc2012/docs/Guarino2009_What_is_an_Ontology.pdf $>$. Acesso em 
dez.2017.

IHMConcept. Map Software a knowledge construction toolkit. Disponível em: $<$ http://www.ihmc.us/> Acesso em fev.2018.

MAYER JOHNSON. (2017) Informações sobre o software Boardmaker e Speaking Dynamically Pro. Disponível em: https://mayer-johnson.com/pages/what-isboardmaker. Acesso mar.2017.

NOY, N. F. \& MCGUINNESS, D. L. (2001) Ontology Development 101: A Guide to Creating Your First Ontology. Stanford Knowledge Systems Lab. Technical Report KSL-01-05 and Stanford Medical Informatics Technical Report SMI-2001-0880. Disponível<protege.stanford.edu/publications/ontology_development/ontology101.pdf> Acesso em jun.2017.

PASSERINO, L. M.; AVILA, B. G.; BEZ, M. R. SCALA: um Sistema de Comunicação Alternativa para o Letramento de Pessoas com Autismo. RENOTE. Revista Novas Tecnologias na Educação, v. 1, p. 1-10, 2010.

PASSERINO, Liliana Maria; BEZ, Maria Rosangela. (2013). Building an Alternative Communication System for literacy of children with autism (SCALA) with Context-Centered Design of Usage. In: Autism / Book 1, v. 1, p. 655-679.

PELOSI, Miryam Bonadiu; BORGES, José Antônio dos Santos.(2015). Prancha Fácil Uma nova abordagem para produção de pranchas para Comunicação Alternativa. Congresso ISAAC Brasil, Campinas, junho 2015. Disponível em < https://docs.google.com/viewer?a=v\&pid=sites\&srcid=bmNlLnVmcmouYnJ8cHJhbmN oYS1mYWNpbHxneDoyYjZkN2NkZDg5MzUzZGNl> Acesso em maio.2018.

USCHOLD, M.; KING, M. (1995). Towards a Methodology for Building Ontologies. In Workshop on Basic Ontological Issues in Knowledge Sharing, in IJCAI-95. Disponível<http://ceur-ws.org/Vol-47/ONTOL2-Proceedings.pdf> Acesso em nov.2017.

VIEYTES, Rut. (2004) Metodología de la investigación em organizaciones, mercado y sociedade: epistemologia y técnica. $1^{\text {a }}$ ed. Buenos Aires: Editorial de las ciências, $732 \mathrm{p}$. 